\title{
A Unified Approach to Approximating Resource Allocation and Scheduling
}

\author{
AMOTZ BAR-NOY \\ AT\&T Shannon Lab, Florham Park, NJ, USA \\ REUVEN BAR-YEHUDA, ARI FREUND AND JOSEPH (SEFFI) NAOR
}

Technion, Isreal Institute of Technology, Haifa, Israel

AND

BARUCH SCHIEBER

IBM T.J. Watson Research Center, Yorktown Heights, NY, USA

Abstract. We present a general framework for solving resource allocation and scheduling problems.
Given a resource of fixed size, we present algorithms that approximate the maximum throughput or
the minimum loss by a constant factor. Our approximation factors apply to many problems, among
which are: (i) real-time scheduling of jobs on parallel machines, (ii) bandwidth allocation for sessions
between two endpoints, (iii) general caching, (iv) dynamic storage allocation, and (v) bandwidth
allocation on optical line and ring topologies. For some of these problems we provide the first constant
factor approximation algorithm. Our algorithms are simple and efficient and are based on the local-
ratio technique. We note that they can equivalently be interpreted within the primal-dual schema. Categories and Subject Descriptors: F.2.2 [Analysis of Algorithms and Problem Complexity]: Nonnumerical Algorithms and Problems-Computations on discrete structures; Sequencing and scheduling

General Terms: Algorithms, Performance

Additional Key Words and Phrases: Approximation algorithms for NP-hard problems, dynamic storage allocation, general caching, resource allocation, scheduling

A preliminary version of this paper appeared in proceedings of the 32nd annual ACM Symposium on Theory of Computing, 2000, pp. 735-744.

The second author was supported by the Technion V.P.R. Fund and the New York Metropolitan Research Fund. The third Author was supported by the Ministry of Trade and Industry's MAGNET program through the CONSIST consortium.

Authors' addresses: A. Bar-Noy, AT\&T Shannon Lab, P. O. Box 971, Florham Park, NJ 07932, on leave from the Electrical Engineering Department, Tel Aviv University, Tel Aviv 69978, Israel, email: amotz@research.att.com; R. Bar-Yehuda, A. Freund, and J.(S.) Naor, Department of Computer Science, Technion, Haifa 32000, Israel, email: \{reuven,arief,naor\}@cs.technion.ac.il; B. Schieber, IBM T.J. Watson Research Center, P.O. Box 218, Yorktown Heights, NY 10598, email: sbar@watson.ibm.com.

Most of this work was done while the second author visited DIMACS during the summer of 1999, and the third and fourth authors were at Bell Laboratories, Lucent Technologies, 600 Mountain Ave., Murray Hill, NJ 07974.

Permission to make digital/hard copy of all or part of this material without fee for personal or classroom use provided that the copies are not made or distributed for profit or commercial advantage, the ACM copyright/server notice, the title of the publication, and its date appear, and notice is given that copying is by permission of the ACM, Inc. To copy otherwise, to republish, to post on servers, or to redistribute to lists requires prior specific permission/or a fee.

(C) 2001 ACM 0004-5411/01/0900-1069 \$5.00 


\section{Introduction}

We study the general problem of resource allocation and activity scheduling. In this problem we have a set of activities competing for a reusable resource. Each activity utilizes a certain amount of the resource for the duration of its execution and frees it upon completion. The problem is to select a feasible subset of activities for execution, that is, a set of activities such that the total amount of resource allocated simultaneously for executing activities never exceeds the amount of resource available. A typical activity may admit several alternatives for its execution, at most one of which is to be selected. Each alternative consists of a time interval during which the activity is to take place, the resource requirement for the activity should it take place in this interval, and the profit to be gained by scheduling the activity in this interval. We allow the length of the time interval, the resource demand, and the profit to vary among different time intervals pertaining to the same activity. Thus, the objective is to find a feasible schedule specifying which activities are selected and when each is to be executed, so as to maximize the profit accrued. We also consider the complement objective of minimizing the profit lost due to unscheduled activities.

This scenario models a wide range of applications. Two basic problems are immediately seen to fit in this framework: bandwidth allocation for sessions in communication networks and machine scheduling of jobs. Another, less obvious, problem that fits in this framework is the general caching problem in which a fixed size cache is used to store pages of varying size and reload cost. Finally, problems such as dynamic storage allocation, in which the resource must be allocated "contiguously," can be cast in our framework by adding a contiguity constraint.

The simplest problem that can be modeled in our framework is the problem of finding a maximum weight independent set in interval graphs [Golumbic 1980]. This problem, which can be solved precisely and efficiently, corresponds to the case where the resource is a single machine and each activity is a task that requires the entire machine for its execution in precisely one time interval. No two tasks may run concurrently, hence feasible schedules correspond to independent sets. Although this problem is polynomial time solvable, it becomes NP-hard if either the resource requirement or the single-time-interval-per-activity constraint are relaxed. If activities may require arbitrary amounts of the resource, the problem is NP-hard since it contains the NP-hard knapsack problem [Garey and Johnson 1979] as a special case (in which all time intervals intersect); if activities are allowed to have multiple time intervals, the problem is known as interval scheduling, which is MaxSNP-hard [Spieksma 1999].

1.1. Previous WORK AND OUR CONTRIBUtion. We provide a unified approach to treating problems formulated in our model by using a novel technique for combining time and resource constraints. Our approach yields constantfactor approximations for all the problems we consider in this paper. For some of them this is the first constant factor approximation algorithm. Our algorithms are based on the local ratio technique [Bar-Yehuda and Even 1985] and they are simple and efficient. We also show how to interpret them within the primal-dual schema. We remark that obtaining a primal-dual algorithm for a (natural) maximization problem was posed recently as an open problem [Vazirani 1999]. Following our work, and that of Berman and DasGupta [2000], 
several authors have applied the same ideas to various auction problems (see, e.g., Akcoglu et al. [2002]).

Following is a list, subdivided into broad categories, of the problems to which we applied our unified approach. We note that the time intervals in which an activity is allowed to be scheduled can be given either as an explicit list (called discrete input), or as a collection of time windows (called continuous input).

Machine Scheduling. The resource consists of $k$ parallel machines and the activities are jobs to be scheduled on these machines. Each job can be scheduled in one of several time intervals. The goal is to maximize the profit of the executed jobs. There are several subcases to this problem.

Maximum Weight Independent Set in Interval Graphs. Here, $k=1$, and each job may be scheduled in one interval only. This problem is well known to be polynomialtime solvable [Golumbic 1980]. Our algorithm also solves it optimally.

Interval Scheduling. Here, $k=1$, and each job may be scheduled in one of a finite set of time intervals. A simple greedy $1 / 2$-approximation algorithm for the unweighted case (where all instances of all activities have identical profit) was given by Spieksma [1999]. A 1/2-approximation factor via linear programming (LP) is implicit in Bar-Noy et al. [2001] for the weighted case (arbitrary profits). Our algorithm achieves the same approximation factor for the weighted case combinatorially. Single Machine Scheduling with Release Times and Deadlines. Again, $k=1$. Each job $i$ has a release time $r_{i}$, a deadline $d_{i}$, and a length $l_{i}$, such that $d_{i} \geq$ $r_{i}+l_{i}$. The job may be scheduled in any interval $\left[x, x+l_{i}\right)$ such that $x$ is in the time window $\left[r_{i}, d_{i}-l_{i}\right]$. Bar-Noy et al. [2001] described an LP-based 1/3approximation algorithm for the weighted case. Our algorithm achieves $(1-\varepsilon) / 2$. It even allows jobs to have multiple time windows (as does the algorithm of Bar-Noy et al. [2001]).

Scheduling on Parallel Unrelated Machines. Here, $k$ is an arbitrary number, and the profit gained by scheduling a job depends both on the job and on the machine to which it is assigned (the input lists the profit corresponding to each job/machine pair). Bar-Noy et al. [2001] demonstrated a greedy 1/2-approximation algorithm for the unweighted case. For the weighted case, they gave an LP-based algorithm that achieves a factor of $1 / 3$ for discrete input and $1 / 4$ for continuous input. Our algorithm achieves $1 / 2$ approximation for discrete input and $(1-\epsilon) / 2$ for continuous input.

Bar-Noy et al. [2001] also treated the case of identical machines, i.e., when the profit of a job is the same for all machines. They presented a greedy algorithm achieving a factor of $1-1 /(1+1 / k)^{k}$ for the unweighted case, and an LP-based algorithm achieving $1-1 /(1+1 /(2 k))^{k}$ for the weighted case (and continuous input). The former expression increases with $k$ from $1 / 2$ to $1-1 / e \approx 0.63$, and the latter increases from $1 / 3$ to $1-1 / \sqrt{e} \approx 0.39$.

Maximum Weight k-Colorable Subgraph in Interval Graphs. This is a special case of the previous problem in which each job may be scheduled in precisely one interval. It can be solved optimally in polynomial time via minimum cost flow [Arkin and Silverberg 1987]. We achieve a $\left(\frac{1}{2}+\frac{1}{4 k-2}\right)$-approximation factor through a simpler and faster algorithm.

All of our algorithms for the problems listed above are in fact instances of a single parameterized algorithm, where the value of the parameter changes 
from problem to problem. The basic algorithm, (i.e., for discrete input) runs in polynomial time, specifically, $\mathrm{O}(n \log n)$ time, where $n$ is the number of instances. To handle time windows efficiently we introduce a certain profit rounding process, which degrades the approximation factor by a factor of $1-\epsilon$. The running time then becomes $\mathrm{O}\left(n^{2} / \epsilon\right)$.

Independent of our work, Berman and DasGupta [2000] developed an algorithm for interval scheduling that is nearly identical to ours. They, too, employ the same rounding idea we use in order to contend with time windows. In addition to single machine scheduling, they also consider scheduling on parallel machines, both identical and unrelated. Here, their approach deviates from ours. Rather than dealing with these problems directly, they solve them via single machine scheduling (for unrelated machines they use a simple reduction to the single machine case, and for identical machines they repeatedly solve single machine instances à-la Bar-Noy et al. [2001]). The approximation factors they achieve are $1-\left(\frac{k+\epsilon}{k+1}\right)^{k}$ for identical machines and $(1-\epsilon) / 2$ for unrelated machines. Finally, they also consider single machine scheduling in the special case where the stretch factors (i.e., $\left.\left(d_{i}-r_{i}\right) / l_{i}\right)$ of all jobs are bounded by some constant $\alpha>2$. Using repeated runs of a modified version of the algorithm they achieve $\frac{1}{2}-\epsilon+1 /\left(2^{a+2}-4-2 a\right)$, where $a=\lfloor\alpha\rfloor$.

Bandwidth Allocation of Sessions in Communication Networks. In modern communication networks (e.g., ATM networks), there exists some available bandwidth between two endpoints in the network. The bandwidth allocation problem is the problem of finding the most profitable set of sessions that can utilize the available bandwidth. Our framework includes this problem, and moreover, we capture the case in which a session may have either a time window or a finite set of intervals in which it can be scheduled. Our algorithm for this problem achieves a factor of $1 / 5$ (or $\frac{1}{5}(1-\epsilon)$ if time windows are allowed). For the special case where each activity consists of a single instance we achieve 1/3. Prior to our work, no constant factor approximation algorithms for this problem were known. Independent of our work, Phillips et al. [2000] obtained approximation algorithms for this problem via LP rounding. For discrete input they achieved an approximation factor of 0.19 and for continuous input they obtained 0.12. (Although their paper states factors of $1 / 4$ and $1 / 6$, respectively, there seems to be an error in the proof. The correct bounds are those given here [Phillips 2001].) We remark that Albers et al. [1999] implies a constant factor approximation (where the constant is about 22) via LP rounding for the special case where each session can be scheduled in precisely one time interval [Khanna 1999].

General Caching. The general caching problem models situations in which a cache is to be used for pages of varying sizes and of varying (cache) reload costs. Specifically, the input consists of a cache size and a sequence of requests for pages. When a page is requested it must be loaded into the cache, unless it is already present there. Each page is characterized by its size and its reload cost, which is incurred whenever the page is loaded into the cache. (Requests for pages in the cache incur no cost.) Since the cache size is fixed, loading one page may necessitate evicting another. The goal is thus to find a minimum cost replacement schedule. We consider the off-line version of this problem, i.e., the case where the input is given ahead of time. As was shown in Albers et al. [1999], the general caching 
problem can be modeled in our framework. Our algorithm for this problem yields a 4-approximation. (Note that since this is a minimization problem the approximation ratio is at least 1.) This improves the results of Albers et al. [1999], who were only able to achieve an $\mathrm{O}(1)$-approximation factor (using LP rounding) by increasing the size of the cache by $\mathrm{O}(1)$ times the largest page size. If the cache size is not increased, then they achieve an $\mathrm{O}(\log (M+C))$ approximation factor, where $M$ and $C$ denote the cache size and the largest page reload cost, respectively.

Contiguous Allocation. Suppose the resource must be allocated in "contiguous" blocks that should remain the same until the resource is freed. For example, in the dynamic storage allocation problem objects are to be stored in computer memory for specified durations. Each object must be allocated a block of contiguous memory, and once the object is stored, the location of its storage block may not be altered. The traditional goal in contiguous allocation has been to store all given objects in minimum size memory [Gergov 1996; 1999; Kierstead 1991]. The throughput version of this problem is, given a fixed size memory, maximize the profit of the objects successfully stored. To the best of our knowledge there is no prior art for the throughput version of the dynamic storage allocation problem. We obtain a $1 / 7$ factor approximation for this problem. We also solve the problem in the general case where each object can be stored during one of several alternative time intervals. For this generalization we get a factor of $1 / 11$ (or $(1-\epsilon) / 11$ for continuous input). Briefly, our approach is to apply our algorithm and solve the non-contiguous version of the problem. We then use the non-contiguous schedule as input to one of the aforementioned memory minimization algorithms. In the final step we extract from the resultant solution - which is contiguous but may exceed the memory limit-a feasible solution that is contiguous and at the same time respects the memory limit. The entire process incurs a constant-factor loss.

Another example of contiguous allocation is strip packing [Steinberg 1997] where the goal is to pack rectangles into a strip. Our constant-factor bounds apply to this problem as well.

Independent of our work, Leonardi et al. [2000] developed an approximation algorithm for the throughput version of the dynamic storage allocation problem. They achieved performance guarantees of $1 / 12$ for discrete input and 1/18 for continuous input, both via LP rounding.

The Line and Ring Topology Networks. Consider the special case of the bandwidth allocation problem where each session can be scheduled in precisely one time interval and the start and end times of every such interval are restricted to be integral time instants. This subproblem can be cast in different terms. Consider a set of processors connected along a line where each processor is identified by its index along this line. By letting the processor identities play the role of the integral time instants in the original problem, we reduce the problem of bandwidth allocation for permanent connections between processors on a line to the bandwidth allocation problem with time intervals defined previously. Clearly, all of our results regarding the time interpretation hold for the processors interpretation as well. They hold for both throughput maximization and loss minimization. An interesting application of our general framework concerns the case where processors are connected along a ring rather than a line. The ring topology is considered a viable network topology in the optical network setting and is well studied in the context of bandwidth allocation. In the ring topology a session between two processors may choose between 
two routes: going clockwise and going counterclockwise. In our framework, this is equivalent to two instances per activity. Most of the prior work in optical networks considered the identical width case in which each session requires one wavelength. Our solution addresses the general case when more than one wavelength per session is available. We show how to adapt any approximation result for the throughput maximization problem on the line into one for the same problem on the ring. Given that the approximation factor for the line is $1 / \rho$, the resulting factor for the ring is $1 /(\rho+1+\epsilon)$, where $\epsilon$ may be chosen arbitrarily small. (The running time of the algorithm depends polynomially on $1 / \epsilon$.)

\section{Preliminaries}

In our general framework the input consists of a set of activities, each requiring the utilization of a given, limited, resource. The amount of resource available is fixed over time; we normalize it to unit size for convenience. (We remark that some of our problems can be generalized to the case where the amount of available resource may change over time.) The activities are specified as a collection of sets $\mathcal{A}_{1}, \ldots, \mathcal{A}_{m}$. Each set represents a single activity: it consists of all possible instances of that activity. An instance $I \in \mathcal{A}_{i}$ is defined by the following parameters.

(1) A half-open time interval $[s(I), e(I))$ during which the activity will be executed. We call $s(I)$ and $e(I)$ the start-time and end-time of the instance.

(2) The amount of resource required for the activity. We refer to this amount as the width of the instance and denote it $w(I)$. (Our terminology is inspired by bandwidth allocation problems.) Naturally, $0<w(I) \leq 1$.

(3) The profit $p(I) \geq 0$ gained by scheduling this instance of the activity.

Different instances of the same activity may have different parameters of duration, width, or profit. A schedule is a collection of instances. It is feasible if: (1) it contains at most one instance of every activity, and (2) for all time instants $t$, the total width of the instances in the schedule whose time interval contains $t$ does not exceed 1 . In the throughput maximization problem we are asked to find a feasible schedule that maximizes the total profit accrued by instances in the schedule. In the loss minimization problem we seek a feasible schedule of minimum penalty, where the penalty of a schedule is defined as the total profit of activities not in the schedule. (We restrict each activity to consist of a single instance and define the profit of an activity to be the profit of its instance.) For a given profit function $p$, we use the term $p$-profit (or $p$-penalty in the loss minimization context) to refer to the profit with respect to $p$ of a single instance or a set of instances.

2.1. The Local Ratio Technique. Our algorithms are based on the local ratio technique, first developed by Bafna et al. [1999], later extended by Bafna, Berman, and Fujito [1999], and recently extended again by Bar-Yehuda [2000]. These papers all treated minimization covering problems.

Let $\mathbf{p} \in \mathbb{R}^{n}$ be a profit (or penalty) vector, and let $F$ be a set of feasibility constraints on vectors $\mathbf{x} \in \mathbb{R}^{n}$. A vector $\mathbf{x} \in \mathbb{R}^{n}$ is a feasible solution to a given problem instance $(F, \mathbf{p})$ if it satisfies all of the constraints in $F$. Its value is the inner product $\mathbf{p} \cdot \mathbf{x}$. A feasible solution is optimal for a maximization (or minimization) problem if its value is maximal (or minimal) among all feasible solutions. A feasible solution $\mathbf{x}$ is an $r$-approximate solution, or simply an $r$-approximation, if $\mathbf{p} \cdot \mathbf{x} \geq$ 
(or $\leq$ ) $r \cdot \mathbf{p} \cdot \mathbf{x}^{*}$, where $\mathbf{x}^{*}$ is an optimal solution. An algorithm is said to have a performance guarantee of $r$ if it always computes $r$-approximate solutions.

In this paper we further extend the local ratio technique to cover problems of the form described above; that is, given a profit (or penalty) vector $\mathbf{p} \in \mathbb{R}^{n}$, find a solution vector $\mathbf{x}$ that maximizes (or minimizes) the inner product $\mathbf{p} \cdot \mathbf{x}$, subject to a given set $F$ of feasibility constraints on $\mathbf{x}$.

THEOREM 2.1 (LOCAL RATIO). Let $F$ be a set of constraints and let $\mathbf{p}, \mathbf{p}_{\mathbf{1}}$, and $\mathbf{p}_{\mathbf{2}}$ be profit (or penalty) vectors such that $\mathbf{p}=\mathbf{p}_{\mathbf{1}}+\mathbf{p}_{\mathbf{2}}$. Then, if $\mathbf{x}$ is an $r$-approximate solution with respect to $\left(F, \mathbf{p}_{\mathbf{1}}\right)$ and with respect to $\left(F, \mathbf{p}_{\mathbf{2}}\right)$, then it is an $r$-approximate solution with respect to $(F, \mathbf{p})$.

ProOF. Let $\mathbf{x}^{*}, \mathbf{x}_{\mathbf{1}}^{*}, \mathbf{x}_{\mathbf{2}}^{*}$ be optimal solutions for $(F, \mathbf{p}),\left(F, \mathbf{p}_{\mathbf{1}}\right)$, and $\left(F, \mathbf{p}_{\mathbf{2}}\right)$ respectively. Then $\mathbf{p} \cdot \mathbf{x}=\mathbf{p}_{\mathbf{1}} \cdot \mathbf{x}+\mathbf{p}_{\mathbf{2}} \cdot \mathbf{x} \geq$ (or $\left.\leq\right) r \cdot \mathbf{p}_{\mathbf{1}} \cdot \mathbf{x}_{\mathbf{1}}^{*}+r \cdot \mathbf{p}_{\mathbf{2}} \cdot \mathbf{x}_{\mathbf{2}}^{*} \geq$ $($ or $\leq) r \cdot\left(\mathbf{p}_{\mathbf{1}} \cdot \mathbf{x}^{*}+\mathbf{p}_{2} \cdot \mathbf{x}^{*}\right)=r \cdot \mathbf{p} \cdot \mathbf{x}^{*}$

The Local Ratio Theorem applies to all problems in the above formulation. Note that $F$ can include arbitrary feasibility constraints and not just linear, or linear integer, constraints. Nevertheless, all successful applications of the local ratio technique to date involve problems in which the constraints are either linear or linear integer, and this is also the case for the problems treated herein.

\section{Throughput Maximization}

In the throughput maximization problem we wish to find a feasible schedule that maximizes the total profit accrued. More formally, the goal is to find an optimal solution to the following integer programming problem on the set of boolean variables $\left\{x_{I} \mid I \in \mathcal{A}_{i}, 1 \leq i \leq n\right\}$.

$$
\text { Maximize } \sum_{I} p(I) \cdot x_{I}
$$

subject to:

for each time instant $t: \sum_{I \mid s(I) \leq t<e(I)} w(I) \cdot x_{I} \leq 1$;

for each activity $\mathcal{A}_{i}$ : $\quad \sum_{I \in \mathcal{A}_{i}} x_{I} \leq 1$;

for each instance $I$ : $\quad x_{I} \in\{0,1\}$.

3.1. The Unified AlgORITHM. We present a generic scheme based on the local ratio technique to approximate the throughput maximization problem. We actually generalize the problem a bit and allow negative profits. Our scheme proceeds as follows.

(1) Delete all instances with non-positive profit.

(2) If no instances remain, return the empty schedule. Otherwise, proceed to the next step.

(3) Select an instance $\tilde{I}$ and decompose $p$ by $p=p_{1}+p_{2}$. The exact choice of $\tilde{I}$ and the decomposition of $p$ depend on the problem at hand and will be discussed 
shortly. (Comment: in the decomposition $p=p_{1}+p_{2}$, the component $p_{2}$ may be non-positive.)

(4) Solve the problem recursively using $p_{2}$ as the profit function. Let $\mathcal{S}^{\prime}$ be the schedule returned.

(5) If $\mathcal{S}^{\prime} \cup\{\tilde{I}\}$ is a feasible schedule, return $\mathcal{S}=\mathcal{S}^{\prime} \cup\{\tilde{I}\}$. Otherwise, return $\mathcal{S}=\mathcal{S}^{\prime}$.

We now analyze the quality of the solution returned by this algorithm. We say that a feasible schedule is $I$-maximal if either it contains instance $I$, or it does not contain $I$ but adding $I$ to it will render it infeasible. We are going to choose $\tilde{I}$ and decompose $p$ in such a way that: (1) $p_{2}(\tilde{I})=0$, and (2) for a certain $r$, which depends on the problem being solved, every $\tilde{I}$-maximal schedule will be an $r$-approximation with respect to $p_{1}$.

PROPOSITION 3.1. Let $r$ be a constant. Suppose that the method for choosing $\tilde{I}$ and decomposing the profit function is such that: $(1) p_{2}(\tilde{I})=0$, and (2) for all profit functions $p$, every $\tilde{I}$-maximal schedule is an $r$-approximation with respect to $p_{1}$. Then, the schedule $\mathcal{S}$ returned by the algorithm is an $r$-approximation.

Proof. Clearly, the first step in which instances of non-positive profit are deleted does not change the optimum value. Thus, it is sufficient to show that $\mathcal{S}$ is an $r$-approximation with respect to the remaining instances. The proof is by induction on the number of recursive calls. At the basis of the recursion, the schedule returned is optimal (and hence an $r$-approximation), since no instances remain. For the inductive step, assume that $\mathcal{S}^{\prime}$ is an $r$-approximation with respect to $p_{2}$. Then, since $p_{2}(\tilde{I})=0$ and $\mathcal{S}^{\prime} \subseteq \mathcal{S} \subseteq \mathcal{S}^{\prime} \cup\{\tilde{I}\}$, it follows that $\mathcal{S}$ is an $r$-approximation with respect to $p_{2}$. Since $\mathcal{S}$ is $\tilde{I}$-maximal, it is also an $r$-approximation with respect to $p_{1}$. Thus, by the Local Ratio Theorem, it is an $r$-approximation with respect to $p$.

It remains to specify how to determine $\tilde{I}$ and the decomposition of the profit function. The choice of $\tilde{I}$ is done by selecting an instance with minimum end-time among all activity instances (of all activities), breaking ties arbitrarily. To define the decomposition $p=p_{1}+p_{2}$, it suffices to define $p_{1}$. We use a parameter $\alpha>0$, whose value we fix for each problem separately, as follows. For a given instance $I$, let $\mathcal{A}(I)$ be the activity to which instance $I$ belongs and let $\mathcal{I}(I)$ be the set of instances intersecting $I$ but belonging to activities other than $\mathcal{A}(I)$. Then,

$$
p_{1}(I)=p(\tilde{I}) \cdot \begin{cases}1 & I \in \mathcal{A}(\tilde{I}), \\ \alpha \cdot w(I) & I \in \mathcal{I}(\tilde{I}), \\ 0 & \text { otherwise. }\end{cases}
$$

Note that $p_{2}(\tilde{I})=0$; hence, $\tilde{I}$ will be deleted in the subsequent recursive call. Thus the algorithm is guaranteed to halt.

The choice of $\alpha$ influences the performance guarantee $r$ we obtain. By Proposition 3.1 we only need to show that every $\tilde{I}$-maximal schedule is an $r$-approximation with respect to $p_{1}$. To do so, we derive an upper bound $b_{\text {opt }}$ on the optimum $p_{1}$-profit and a lower bound $b_{\max }$ on the $p_{1}$-profit of every $\tilde{I}$-maximal schedule, both normalized by $p(\tilde{I})$, which is to say that the actual bounds are $p(\tilde{I}) \cdot b_{\max }$ and $p(\tilde{I}) \cdot b_{\text {opt }}$. The ratio $r=b_{\max } / b_{\text {opt }}$ is then a lower bound on the performance of the algorithm.

We now derive formulas for $b_{\text {opt }}$ and $b_{\max }$. These formulae are valid for the generic problem and thus for all special cases as well. However, for specific problems 
within the framework we are sometimes able to show better choices of $b_{\text {opt }}$ and $b_{\max }$ (i.e., tighter bounds that result in better performance guarantees).

Consider an optimal schedule. By the definition of $p_{1}$, only instances in $\mathcal{A}(\tilde{I}) \cup$ $\mathcal{I}(\tilde{I})$ contribute to its $p_{1}$-profit. Since all the instances belonging to $\mathcal{I}(\tilde{I})$ intersect at some point infinitesimally close to $e(\tilde{I})$, the total width of such instances in the optimal schedule can be at most 1 , and their contribution to the $p_{1}$-profit is therefore at most $\alpha \cdot p(\tilde{I})$. The contribution of the instances in $\mathcal{A}(\tilde{I})$ is at most $p(\tilde{I})$ since a feasible schedule may contain at most one instance of each activity. Thus, we have a bound $b_{\mathrm{opt}}=1+\alpha$.

Turning to $\tilde{I}$-maximal schedules, observe that every such schedule either contains an instance of $\mathcal{A}(\tilde{I})$ or else contains a set $\mathcal{X} \neq \emptyset$ of instances intersecting $\tilde{I}$ that prevent $\tilde{I}$ from being added to the schedule. In the former case the $p_{1}$-profit of the schedule is at least $p(\tilde{I})$. In the latter case, we can bound the profit from below as follows. Let $w_{\max }$ and $w_{\min }$ be upper and lower bounds, respectively, on the width of instances in the input. Then the total width of instances in $\mathcal{X}$ is at least $1-w(\tilde{I}) \geq 1-w_{\max }$, for otherwise $\tilde{I}$ can be added without violating the feasibility of the schedule, and it is also not less than $w_{\min }$ since $\mathcal{X} \neq \emptyset$. Since $\tilde{I}$ was chosen as an instance with minimum end-time, every instance $I$ that intersects it must satisfy $s(I)<e(\tilde{I}) \leq e(I)$. Hence, $\mathcal{X} \subseteq \mathcal{I}(\tilde{I})$, and the $p_{1}$-profit of the schedule is at least $\alpha \cdot p(\tilde{I}) \cdot \max \left\{w_{\min }, 1-w_{\max }\right\}$. Thus, we can use the bound $b_{\max }=\min \left\{1, \alpha \cdot \max \left\{w_{\min }, 1-w_{\max }\right\}\right\}$. In the general case, this bound is meaningless, since $w_{\min }$ and $1-w_{\max }$ may both be arbitrarily close to 0 . However, the various problems we treat impose restrictions on the allowable widths of instances, and we can obtain meaningful bounds.

LEMMA 3.2. The approximation factor of the algorithm is at least $b_{\max } / b_{\text {opt }}$ for all provable bounds $b_{\max }$ and $b_{\text {opt }}$, and $b_{\max }$ and $b_{\text {opt }}$ can always be chosen such that this ratio is at least

$$
\frac{\min \left\{1, \alpha \cdot \max \left\{w_{\min }, 1-w_{\max }\right\}\right\}}{1+\alpha} .
$$

3.2. APPLiCATiOns. The throughput maximization problem generalizes several known problems. In this section we present a selection of these problems and show how to approximate them using our scheme.

In some of the applications described below, an activity $\mathcal{A}$ is not represented by explicitly listing all the instances belonging to it, but rather by a collection of time windows. A time window $\mathcal{T}$ is a time interval $[s(\mathcal{T}), e(\mathcal{T}))$ or $[s(\mathcal{T}), e(\mathcal{T})]$ accompanied by three parameters: length $l(\mathcal{T})$, width $w(\mathcal{T})$, and profit $p(\mathcal{T})$. These are interpreted as follows. Every $t \in \mathcal{T}$ defines an instance with time interval $[t, t+l(\mathcal{T}))$, and all of these instances share the same width $w(\mathcal{T})$ and profit $p(\mathcal{T})$. We stress that the term time window refers to the interval of time in which the activity may begin execution; it is not the time interval spanning the release time and deadline of the activity (these two intervals differ by $l(\mathcal{T})$ ). Note that a time window must be closed on the left but may be closed or open on the right.

This sort of continuous input leads, of course, to problems in the implementation of the algorithm. We discuss this, as well as other implementation issues, in Section 3.3. For the time being, let us just mention that the algorithm can be implemented so that it always returns an $r(1-\epsilon)$-approximation, where $r$ is the performance guarantee that could be achieved had the instances been specified 
explicitly (i.e., discrete input), and $\epsilon>0$ is an arbitrarily small constant. The running time of this implementation is polynomial in $1 / \epsilon$ and the number of time windows in the input.

3.2.1. Single Machine Scheduling. Here we assume that the resource is a single machine and each activity instance requires the machine. This is modeled by the condition $w(I)=1$ for all instances $I$. The following variants of the problem are considered.

Maximum Weight Independent Set in Interval Graphs. Consider the case in which each activity $\mathcal{A}_{i}$ is a singleton $\left\{I_{i}\right\}$. This is exactly the problem of finding a maximum-weight independent set in an interval graph, where each instance $I_{i}$ corresponds to an interval. This is a well known problem, which can be solved optimally and efficiently by reducing it to the problem of finding the longest path in a DAG [Golumbic 1980]. We claim that the unified algorithm with $\alpha=1$ yields an optimal solution. To see this, observe that since $\mathcal{A}(\tilde{I})=\{\tilde{I}\}$, only one instance from $\mathcal{A}(\tilde{I}) \cup \mathcal{I}(\tilde{I})$ may be scheduled in any feasible solution, hence $b_{\text {opt }}=1$. In addition, $w_{\min }=w_{\max }=1$, so $b_{\max }=1$. Thus the performance guarantee is $b_{\max } / b_{\text {opt }}=1$.

Interval Scheduling. In this problem, each activity consists of a set of instances that are explicitly specified (see, e.g., Erlebach and Jansen [1998, 2000]; Spieksma [1999]). This time we use the general bound $b_{\text {opt }}=1+\alpha$ and set $\alpha=1$ so as to maximize Expression 1, given that $w_{\min }=w_{\max }=1$. By Lemma 3.2 the performance guarantee is $1 / 2$.

Maximum Weight Throughput. Here activities are specified as time windows. This is exactly the problem of maximizing the weighted throughput of jobs with release times and deadlines on a single machine considered in Bar-Noy et al. [2001]. Since the only difference between this problem and the previous one is that that the instances are not listed explicitly, we get a performance guarantee of $(1-\epsilon) / 2$.

3.2.2. Parallel Machine Scheduling. Here we assume that the resource is a set of $k$ parallel machines. There are two sub-cases to this problem: identical machines, where each activity instance may be assigned to any of the $k$ machines, and unrelated machines, where each activity instance specifies a particular machine on which it may be scheduled. (In case an instance may be scheduled on several machines we simply replicate the instance once for each machine.)

Note that our general framework does not capture this problem completely, since it does not model the condition that every activity must be carried out in its entirety on a single machine. We now argue that the solution returned by our algorithm will never force us to split an activity among several machines. This is clearly the case for unrelated machines since in this sub-case each activity instance specifies a single machine. In the case of identical machines, given a schedule returned by our algorithm, we consider the activity instances in the schedule in non-decreasing order of their start-times, and assign each activity to an arbitrary available machine. The feasibility of the schedule guarantees that at least one machine will always be available.

Identical Machines. We model this case by setting the width of every instance equal to $1 / k$. We use $\alpha=1$ and $b_{\text {opt }}=1+\alpha=2$. In addition, we can take $b_{\max }=1$ since any $\tilde{I}$-maximal schedule that contains no instance of $\mathcal{A}(\tilde{I})$ must contain $k$ 
instances from $\mathcal{I}$. Thus we get a performance guarantee of $1 / 2$ (or $(1-\epsilon) / 2$ if the activities are specified as time windows).

A special case of interest is maximum weight $k$-colorable subgraph in interval graphs. This is just the case of identical machines with each activity consisting of a single instance. It can be solved optimally in polynomial time via minimum cost flow [Arkin and Silverberg 1987]. Our approach provides a 1/2-approximation factor through a much simpler and faster algorithm. As a matter of fact, the approximation factor for this case is $\frac{1}{2-1 / k}$, since each activity consists of a single instance and thus we can take $b_{\mathrm{opt}}=1+(k-1) / k$.

Unrelated Machines. We model this problem by the condition $w(I)=1$ for all instances $I$, and modify the definition of $\mathcal{I}(\tilde{I})$ as follows. We define $\mathcal{I}(\tilde{I})$ as the set of instances intersecting $\tilde{I}$ that belong to other activities and can be scheduled only on the same machine as $\tilde{I}$. In addition, we use the following criterion in the schedule construction phase. An instance may be added to the schedule if the activity to which it belongs is not currently represented in the schedule and the instance does not intersect any other instance already in the schedule that belongs on the same machine. It is easy to see that the analysis for the case of single machine scheduling carries over to this problem and thus we have a performance guarantee of $1 / 2$ (or $(1-\epsilon) / 2$ for time windows).

3.2.3. Bandwidth Allocation. We consider a scenario in which the bandwidth of a communication channel must be allocated to sessions. Here the resource is the channel's bandwidth and the activities are sessions to be routed through the channel. The sessions may be specified in either of two ways: (1) discrete input that lists for each call a set of intervals in which it can be scheduled, together with a width requirement and a profit for each such interval, or (2) continuous input that uses time windows.

Suppose all instances are narrow, i.e., have width at most $1 / 2$. Then, we use $\alpha=2$ so as to maximize Expression 1, given that $w_{\max } \leq 1 / 2$ and $w_{\min }=0$. Hence, Lemma 3.2 provides a performance guarantee of $1 / 3$ (or $(1-\epsilon) / 3$ in the continuous case).

Next, suppose all instances are wide, i.e., have weight greater than $1 / 2$. Then the problem reduces to interval scheduling (or, in the case of continuous input, to maximum weight throughput on a single machine) since no pair of intersecting instances may be scheduled together. Thus, we have a performance guarantee of $1 / 2$ (or $(1-\epsilon) / 2)$.

Finally, to solve the problem in the general case we solve it separately for the narrow instances and for the wide instances, and return the solution of greater profit. Since either the optimum for the narrow instances is at least three fifths of the optimum (for the original problem), or the optimum for the wide jobs is at least two fifths of the optimum, the schedule returned is a $1 / 5$-approximation (or $(1-\epsilon) / 5)$.

We remark that in the special case in which each activity has only one instance we can obtain a performance guarantee of 1 for the wide instances (since the problem reduces to finding a maximum-weight independent set in an interval graph), and $1 / 2$ for the narrow instances (and therefore $1 / 3$ overall) by choosing $\alpha=1 /(1-w(\tilde{I}))$. This is so because in this special case the $p_{1}$-profit of any $\tilde{I}$-maximal solution is at least $p(\tilde{I}) \cdot \min \{1, \alpha(1-w(\tilde{I}))\}$, whereas the optimal $p_{1}$-profit is at most 
$p(\tilde{I}) \cdot \max \{\alpha, 1+\alpha(1-w(\tilde{I}))\}$. Note that $\alpha$ now depends on $\tilde{I}$, which means a different value is used at different stages in the algorithm. This has no bearing on the correctness of our analysis.

As with machine scheduling, we can extend this problem to $k$ unrelated channels, possibly with different widths, such that each activity instance may only be assigned to one particular channel. The method of solution and the approximation factors carry over from the single channel case.

3.3. IMPLEMENTATION ISSUES. Observe that the algorithm may be implemented iteratively rather than recursively. At each iteration we delete all instances with non-positive profit; find $\tilde{I}, \mathcal{A}(\tilde{I})$, and $\mathcal{I}(\tilde{I})$; compute $p_{1}(I)$ and perform $p(I) \leftarrow p(I)-p_{1}(I)$ for all $I \in \mathcal{A}(\tilde{I}) \cup \mathcal{I}(\tilde{I})$; and push $\tilde{I}$ onto a stack. We iterate until no instances remain. We then construct the schedule by popping the activity instances off the stack and adding each to the current schedule if doing so does not violate the feasibility of the schedule.

A straightforward implementation of this algorithm (when instances are given explicitly and not as time windows) runs in $\Theta\left(n^{2}\right)$ time, where $n$ is the number of instances. The bottleneck is the need to update the profits of the instances in $\mathcal{A}(\tilde{I}) \cup \mathcal{I}(\tilde{I})$ : in the worst case the $i$ th iteration updates the profits of $n-i$ instances. The determination of $\tilde{I}$ and $\mathcal{I}(\tilde{I})$ also requires $\Theta\left(n^{2}\right)$ time, as does the construction of the schedule.

A closer look at the algorithm reveals that the sole purpose of the stack is to reverse the order in which instances are considered in the schedule construction phase. What the algorithm really does is an initial phase, in which it scans the instances in non-decreasing end-time order, deleting some of them as it goes along, and a second phase, in which it scans the surviving instances in reverse order and constructs the schedule. Rather than using a stack, we can accomplish this by sorting the instances in a list and traversing it in both directions. This idea is only one step shy of the more powerful sweep-line approach, which can reduce the worst case time complexity to $\mathrm{O}(n \log n)$, as we now describe.

The time interval of each instance has two endpoints. We denote the instance to which endpoint $t$ belongs by $I(t)$. We sort the $2 n$ endpoints of the instances in a list $\left\langle t_{1}, t_{2}, \ldots, t_{2 n}\right\rangle$ such that $i<j$ if $t_{i}<t_{j}$ or if $t_{i}=t_{j}$ and $t_{i}$ is the end-time of $I\left(t_{i}\right)$ and $t_{j}$ is the start-time of $I\left(t_{j}\right)$. (Otherwise, ties are broken arbitrarily.) We then consider the endpoints in order, reducing profits as we go along and deleting an instance (i.e., deleting its two endpoints from the list) whenever its profit is found to be non-positive. Note that profits never increase, so there is no need to delete the instance the moment its profit drops to zero or less. Instead, we wait with the deletion until the end-time of the instance is encountered.

The pseudo-code below implements the sweep-line idea (though it does not achieve the promised $\mathrm{O}(n \log n)$ time complexity). For each instance $I$ it uses a variable $\pi_{I}$ in which it holds the current profit of $I$. The value of $\pi_{I}$ is maintained correctly only as long as $I$ is alive, that is, as long as $I$ would still be part of the input in the recursive algorithm. To avoid confusion we stress that $p(I)$ denotes the original profit of instance $I$ given in the input, as opposed to $\pi_{I}$ which is the "current" profit.

1. Do $\pi_{I} \leftarrow p(I)$ for all $I$, as well as other initializations.

2. Sort the endpoints as described above.

3. For $i \leftarrow 1,2, \ldots, 2 n$ : 
4. $\quad I \leftarrow I\left(t_{i}\right)$.

5. If $t_{i}$ is the start-time of $I$ : do nothing.

6. $\quad$ Else $\left(t_{i}\right.$ is the end-time of $\left.I\right)$ :

7. $\quad P \leftarrow \pi_{I}$.

8. $\quad$ If $P \leq 0$ : delete $I$.

9. Else:

10. $\quad$ For all $J \in \mathcal{A}(I): \pi_{J} \leftarrow \pi_{J}-P$.

11. $\quad$ For all $J \in \mathcal{I}(I): \pi_{J} \leftarrow \pi_{J}-\alpha \cdot w(J) \cdot P$.

The updating of profits still takes $\Theta\left(n^{2}\right)$ time in the worst case. We can reduce this to linear time by representing the profit function implicitly instead of using the variables $\pi_{I}$. (The initial sorting still requires $\Theta(n \log n)$ time.) With each activity $\mathcal{A}$ we associate a variable $\Delta_{\mathcal{A}}$ and with each instance $I$ we associate a variable $\Delta_{I}$. In addition, we maintain a variable $\Delta_{P}$. All of these variables are initially set to 0 . We represent the profits as follows. The current profit of a live instance $I \in \mathcal{A}$ is $p(I)-\Delta_{\mathcal{A}}$ if neither endpoint of $I$ has been encountered, and it is $p(I)-\Delta_{\mathcal{A}}-\alpha \cdot w(I)\left(\Delta_{P}-\Delta_{\mathcal{A}}\right)+\Delta_{I}$ if its start-time has already been seen but its end-time has not. We modify the algorithm as follows. We replace "do nothing" in Line 5 with $\Delta_{I} \leftarrow \alpha \cdot w(I)\left(\Delta_{P}-\Delta_{\mathcal{A}(I)}\right)$ and we replace: Line 7 by $P \leftarrow p(I)-\Delta_{\mathcal{A}(I)}-\alpha \cdot w(I)\left(\Delta_{P}-\Delta_{\mathcal{A}(I)}\right)+\Delta_{I} ;$ Line 10 by $\Delta_{\mathcal{A}(I)} \leftarrow \Delta_{\mathcal{A}(I)}+P ;$ and Line 11 by $\Delta_{P} \leftarrow \Delta_{P}+P$. This scheme of representing profits works when $\alpha$ is fixed. It can be easily modified to handle the case where $\alpha$ may change (as in our $1 / 2$-approximation algorithm for bandwidth allocation in the special case where each activity consists of a single narrow instance).

When the traversal of the list is complete, we traverse the surviving endpoints in reverse order and construct the schedule $\mathcal{S}$. We maintain a variable $W$ and the following invariant: when endpoint $t$ is reached, $W$ holds the total width of instances containing $t$ which have been added to $\mathcal{S}$.

12. $\mathcal{S} \leftarrow \emptyset$.

13. $W \leftarrow 0$.

14. Traverse the list backwards. For each endpoint $t$ :

15. $\quad I \leftarrow I(t)$.

16. If $t$ is the end-time of $I$ :

17. If $W+w(I) \leq 1$ :

18. $\quad \mathcal{S} \leftarrow \mathcal{S} \cup\{I\}$.

19. $\quad W \leftarrow W+w(I)$.

20. Delete the endpoints of the instances in $\mathcal{A}(I)-\{I\}$.

21. $\quad$ Else: delete the endpoints of $I$.

22. $\quad$ Else $(t$ is the start-time of $I): W \leftarrow W-w(I)$.

3.3.1. Time Windows. In this section we refer to the straightforward stack-based iterative implementation of the algorithm described above. We use $p(\cdot)$ to denote the original profits and $\pi(\cdot)$ to denote the profits as they change throughout the execution of the algorithm.

When the input is in the form of time windows, we do not modify the profit of individual instances, but rather operate on whole windows at a time. At each iteration we delete all windows whose instances have non-positive profit, and find an instance $\tilde{I}$ with earliest end-time among the remaining instances by considering $s(\mathcal{T})+l(\mathcal{T})$ for all remaining windows $\mathcal{T}$. We push $\tilde{I}$ on the stack and update the profits of the instances in $\mathcal{A}(\tilde{I}) \cup \mathcal{I}(\tilde{I})$. Note that to update the profits of instances 
in $\mathcal{I}(\tilde{I})$ and still maintain the property that the profits of all instances in the same time window are the same, we might need to first split some windows, as follows. For each window $\mathcal{T}$ containing instances in $\mathcal{I}(\tilde{I})$, if $e(\mathcal{T})<e(\tilde{I})$, or $e(\mathcal{T})=e(\tilde{I})$ and $\mathcal{T}$ is open, we simply reduce $\pi(\mathcal{T})$. Otherwise, we split $\mathcal{T}$ into two windows $\mathcal{T}^{\prime}=[s(\mathcal{T}), e(\tilde{I}))$ and the remainder $\mathcal{T}^{\prime \prime}=\mathcal{T}-\mathcal{T}^{\prime}$, and reduce $\pi\left(\mathcal{T}^{\prime}\right)$. We refer to this implementation as the precise algorithm. It is easy to see that the points at which the time windows may be split are all of the form: start-time of some window plus a finite sum of lengths of instances (not necessarily of the same activity). Since no such point can be greater than the maximum end-time of an instance (taken over all instances of all activities), there are only finitely many such points. Thus, the precise algorithm always halts in finite, if super-polynomial, time.

In order to attain polynomial running time we trade accuracy for speed. For any fixed $0<\epsilon<1$ we modify the algorithm as follows. Whenever $\tilde{I}$ is chosen such that $0<\pi(\tilde{I})<\epsilon p(\tilde{I})$ we simply delete the window containing $\tilde{I}$ and do not alter any profits. We call such an iteration empty. We refer to this algorithm as the profit truncating algorithm.

Let us analyze the running time of the profit truncating algorithm. We denote by $n$ the number of windows in the input and introduce the following terminology. When a window $\mathcal{T}$ is split into $\mathcal{T}^{\prime}$ and $\mathcal{T}^{\prime \prime}$ we say that one new window is created. We consider the original windows (in the input) to have been created initially. Also, for a given iteration, we say that $\tilde{I}$ is selected in that iteration. Recall that time windows are split in such a way that the profits of all instances in the same window are the same. Hence, the algorithm deletes at least one window in each iteration, so the number of iterations is bounded by the number of windows ever created. Because of the manner in which new windows are created, it is obvious that any time instant may belong to at most $n$ different windows, and thus the number of new windows created in the course of any single iteration is at most $n-1$. To bound the number of windows ever created we therefore bound the number of non-empty iterations, since new windows can only be created in these iterations. Consider an original time window $\mathcal{T}$ given in the input. How many times can the algorithm select an instance belonging (originally) to $\mathcal{T}$ in non-empty iterations? Each time it does so, $\pi(\tilde{I}) \geq \epsilon p(\tilde{I})=\epsilon p(\mathcal{T})$, since the iteration is non-empty. During the iteration the profits of all instances in $\mathcal{A}(\tilde{I})$ decrease by $\pi(\tilde{I})$. In particular, the profit of every surviving instance originally belonging to $\mathcal{T}$ drops by no less than $\epsilon p(\mathcal{T})$. Thus, at most $1 / \epsilon$ such iterations may occur. It follows that the total number of non-empty iterations is at most $n / \epsilon$. Hence, the number of windows ever created is at most $n+n(n-1) / \epsilon<n^{2} / \epsilon$ and this bounds the total number of iterations. The algorithm can be implemented such that the amortized time complexity of an iteration is $\mathrm{O}(1)$ if it is empty and $\mathrm{O}(n)$ if it is not, resulting in an overall time complexity of $\mathrm{O}\left(n^{2} / \epsilon\right)$ for the first phase. Since only non-empty iterations push instances on the stack, and precisely one instance is pushed in each such iteration, the size of the stack cannot exceed $n / \epsilon$. Thus a straightforward implementation of the second phase also requires $\mathrm{O}\left(n^{2} / \epsilon\right)$ time, since the size of the schedule is never greater than $n$. Thus, the time complexity of the entire algorithm is $\mathrm{O}\left(n^{2} / \epsilon\right)$.

We now analyze how the profit truncation affects the performance guarantee. For a given input, consider the time windows that the algorithm deletes in the course of its execution. Every deleted window is derived, by zero or more splitting operations, from a window originally present in the input. Without loss of generality let us assume that the algorithm never splits any windows (for we can always partition 
each window into its constituent deleted windows before commencing execution). We say that window $\mathcal{T}$ is bad if the algorithm deletes it in an empty iteration. Let $\epsilon_{\mathcal{T}}$ be the value of $\pi(\mathcal{T})$ when bad window $\mathcal{T}$ is selected. Consider the following two scenarios. In the first scenario we run the profit truncating algorithm on the original input, and in the second scenario we change the profit of each bad window $\mathcal{T}$ to $p(\mathcal{T})-\epsilon_{\mathcal{T}}$ and then run the precise algorithm. It is easy to see that the precise algorithm in the second scenario can be made to run identically as the profit truncating algorithm in the first, except that at all times the profit of every undeleted bad window $\mathcal{T}$ in the first scenario will be higher by $\epsilon_{\mathcal{T}}$ than its profit in the second scenario. It follows that the schedule $\mathcal{S}$ obtained in the first scenario can also be obtained in the second. Let $p_{\text {opt }}$ and $p_{\text {opt }}^{\prime}$ denote the optimum profit in the first and second scenarios, respectively, and for every feasible schedule $\mathcal{X}$, let $p(\mathcal{X})$ and $p^{\prime}(\mathcal{X})$ be the profits of $\mathcal{X}$ in both scenarios, respectively. Then, by construction, $p(\mathcal{X}) \geq p^{\prime}(\mathcal{X}) \geq(1-\epsilon) p(\mathcal{X})$ for all feasible schedules $\mathcal{X}$. It follows that $p_{\text {opt }}^{\prime} \geq(1-\epsilon) p_{\text {opt }}$. Let $r$ be a performance guarantee for the precise algorithm. Then $p(\mathcal{S}) \geq p^{\prime}(\mathcal{S}) \geq r \cdot p_{\text {opt }}^{\prime} \geq r(1-\epsilon) p_{\text {opt }}$, and thus we have a performance guarantee of $r(1-\epsilon)$ for the profit truncating algorithm.

\section{Loss Minimization}

In the loss minimization problem the objective is to minimize the profit lost to unscheduled activities. In this context, we find it more appropriate to refer to penalty incurred rather than to profit lost. Hence, we associate a penalty with each activity, and our objective is to find a feasible schedule of minimum penalty, where the penalty of a schedule is the sum of penalties of the activities not in the schedule. Our framework cannot model this type of penalty function in general. It can only model it in the special case where each activity contains a single instance. We therefore restrict the discussion to this case.

We actually solve a generalization of the problem in which the amount of resource may vary with time. Thus, the input consists of the activity specification as well as a positive function Width $(t)$ specifying the width (i.e., amount of resource) available at every time instant $t$. Accordingly, we allow arbitrary positive instance widths (rather than assuming that all widths are bounded by 1). A schedule is feasible if for all time instants $t$, the total width of instances in the schedule containing $t$ is at most $\operatorname{Width}(t)$.

Strictly speaking, for the problem to fit in the framework of the Local Ratio Theorem, we should define feasible solutions to be complements of feasible schedules. We find it easier, though, to keep thinking of schedules as solutions and we retain this view in the following exposition.

We present a variant of the unified algorithm achieving a performance guarantee of 4 . Let $(\mathcal{H}, p)$ denote the input, where $\mathcal{H}$ is the description of the activities excluding the penalties, and $p$ is the penalty function. If all the instances in $\mathcal{H}$ constitute a feasible schedule, we return this schedule. Otherwise, we decompose $p$ by $p=p_{1}+p_{2}$ (as described in the next paragraph) such that $p_{2}(I) \geq 0$ for all instances $I$, with equality for at least one instance. We solve recursively $\left(\mathcal{H}^{\prime}, p_{2}\right)$, where $\mathcal{H}^{\prime}$ is obtained from $\mathcal{H}$ by deleting all instances whose $p_{2}$-penalty is 0 , and obtain a schedule $\mathcal{S}^{\prime}$. We then consider the instances in $\mathcal{H}-\mathcal{H}^{\prime}$ in arbitrary order and add each to the schedule iff doing so retains the feasibility of the schedule. We return the schedule $\mathcal{S}$ thus constructed. 
Let us define the decomposition of $p$ by showing how to compute $p_{1}$. For a given time instant $t$, let $\mathcal{I}(t)$ be the set of intervals containing $t$. Define $\Delta(t)=\sum_{I \in \mathcal{I}(t)} w(I)-\operatorname{Width}(t)$. To compute $p_{1}$, find $t^{*}$ maximizing $\Delta(\cdot)$ and let $\Delta^{*}=\Delta\left(t^{*}\right)$. Assuming $\Delta^{*}>0$ (otherwise the schedule containing all instances is feasible), let

$$
p_{1}(I)=\rho \cdot\left\{\begin{array}{cl}
\min \left\{\Delta^{*}, w(I)\right\} & I \in \mathcal{I}\left(t^{*}\right), \\
0 & \text { otherwise, }
\end{array}\right.
$$

where $\rho$ (which depends on $t^{*}$ ) is the unique scaler resulting in non-negative $p_{2}$-penalties for all instances and zero $p_{2}$-penalty for at least one of them. A straightforward implementation of this algorithm runs in time polynomial in the number of activities and the number of time instants at which Width $(t)$ changes value.

To analyze the algorithm, assume (by induction) that $\mathcal{S}^{\prime}$ is a 4-approximation for $\left(\mathcal{H}^{\prime}, p_{2}\right)$. It follows that $\mathcal{S}$ is a 4-approximation for $\left(\mathcal{H}, p_{2}\right)$. Observe (by induction on the recursion) that $\mathcal{S}$ is maximal in the sense that adding any instance to it violates its feasibility. Thus, by the Local Ratio Theorem, it suffices to show that every maximal feasible schedule is a 4-approximation for $\left(\mathcal{H}, p_{1}\right)$.

OBSERVATION 4.1. Both of the following evaluate to at most $\rho \Delta^{*}$ : (1) the $p_{1}$-penalty of any single instance, and (2) the total $p_{1}$-penalty of any collection of instances whose total width is at most $\Delta^{*}$. Also, any collection of instances from $\mathcal{I}\left(t^{*}\right)$ whose total width is at least $\Delta^{*}$ has a total $p_{1}$-penalty of at least $\rho \Delta^{*}$.

Consider an optimal schedule. Its $p_{1}$-penalty is the sum of the $p_{1}$-penalties of the instances in $\mathcal{I}\left(t^{*}\right)$ that are not in the schedule. Since all of these instance intersect at $t^{*}$ their combined width must be at least $\Delta^{*}$. Thus, the optimal $p_{1}$-penalty is at least $\rho \Delta^{*}$. Now consider any maximal feasible solution $\mathcal{M}$. We claim that it is a 4-approximation because its $p_{1}$-penalty cannot exceed $4 \rho \Delta^{*}$. To demonstrate this, consider any time instant $t$. Let $\overline{\mathcal{M}}(t)=\mathcal{I}(t)-\mathcal{M}$ be the set of instances containing $t$ that are not in the schedule. We say that $t$ is unstable if there is an interval $I \in \overline{\mathcal{M}}(t)$ such that adding $I$ to $\mathcal{M}$ would violate the width constraint at $t$. We say that $t$ is unstable because of $I$. Note that a single time instant may be unstable because of several different instances.

LEMMA 4.2. If $t$ is an unstable time instant, then $\sum_{I \in \overline{\mathcal{M}}(t)} p_{1}(I)<2 \rho \Delta^{*}$.

Proof. Let $J$ be an instance of maximum width in $\overline{\mathcal{M}}(t)$. Then, since $t$ is unstable, it is surely unstable because of $J$. This implies that $\sum_{I \in \mathcal{M} \cap \mathcal{I}(t)} w(I)>\operatorname{Width}(t)-w(J)$. Thus, $\sum_{I \in \overline{\mathcal{M}}(t)} w(I)=\sum_{I \in \mathcal{I}(t)} w(I)-$ $\sum_{I \in \mathcal{M} \cap \mathcal{I}(t)} w(I)<w(J)+\Delta(t) \leq w(J)+\Delta^{*}$. Hence, $\sum_{I \in \overline{\mathcal{M}}(t)} p_{1}(I) \leq \rho \Delta^{*}+$ $\rho \Delta^{*}=2 \rho \Delta^{*}$.

Thus, there are two cases to consider. If $t^{*}$ is an unstable point, then the $p_{1}$ penalty of the schedule is $\sum_{I \in \overline{\mathcal{M}}\left(t^{*}\right)} p_{1}(I)<2 \rho \Delta^{*}$ and we are done. Otherwise, let $t_{L}<t^{*}$ and $t_{R}>t^{*}$ be the two unstable time instants closest to $t^{*}$ on both sides (it may be that only one of them exists). The maximality of the schedule implies that every instance in $\overline{\mathcal{M}}\left(t^{*}\right)$ is the cause of instability of at least one time instant. Thus each such instance must contain $t_{L}$ or $t_{R}$ (or both). It follows that $\overline{\mathcal{M}}\left(t^{*}\right) \subseteq \overline{\mathcal{M}}\left(t_{L}\right) \cup \overline{\mathcal{M}}\left(t_{R}\right)$. Hence, by Lemma 4.2 , the total $p_{1}$-penalty of these instances is less than $4 \rho \Delta^{*}$. 
4.1. APPLICATION: GENERAL CACHING. In the general caching problem, a replacement schedule is sought for a cache that must accommodate pages of varying sizes. The input consists of a fixed cache size $S>0$, a collection of pages $\{1,2, \ldots, m\}$, and a sequence of $n$ requests for pages. Each page $j$ has a size $0<s(j) \leq S$ and a cost $c(j) \geq 0$ associated with loading it into the cache. We assume for convenience that time is discrete and that the $i$ th request is made at time $i$. (These assumptions cause no loss of generality as will become evident from our solution.) We denote by $r(i)$ the page being requested at time $i$. A replacement schedule is a specification of the contents of the cache at all times. It must satisfy the following condition. For all $1 \leq i \leq n$, page $r(i)$ is present in the cache at time $i$ and the sum of sizes of the pages in the cache at that time is not greater than the cache size $S$. The initial contents of the cache (at time 0 ) may be chosen arbitrarily. Alternatively, we may insist that the cache be empty initially. The cost of a given replacement schedule is $\sum c(r(i))$ where the sum is taken over all $i$ such that $r(i)$ is absent from the cache at time $i-1$. The objective is to find a minimum cost replacement schedule.

Observe that if we have a replacement schedule that evicts a certain page at some time between two consecutive requests for it, we may as well evict it immediately after the first of these requests and bring it back only for the second request. Thus, we may restrict our attention to schedules in which for every two consecutive requests for a page, either the page remains present in the cache at all times between the first request and the second, or it is absent from the cache at all times in between. This leads naturally to a description of the problem in terms of time intervals, and hence to a straightforward reduction of the problem to our loss minimization problem. This reduction was described in Albers, Arora, and Khanna [1999]; we recount it here for the sake of completeness. Given an instance of the general caching problem we construct an instance of our loss minimization problem as follows. We define the resource width by Width $(i)=S-s(r(i))$ for $1 \leq i \leq n$, and $\operatorname{Width}(0)=S$ (or Width(0) $=0$ if we want the cache to be empty initially). We define the activity instances as follows. Consider the request made at time $i$. Let $j$ be the time at which the previous request for $r(i)$ is made, or $j=-1$ if no such request is made. If $j+1 \leq i-1$, we define an activity instance with time interval $[j+1, i-1]$, penalty $c(r(i))$, and width $s(r(i))$. This reduction implies a 4approximation algorithm for the general caching problem via our 4-approximation algorithm for the loss minimization problem.

\section{Contiguous Allocation of a Non-Fungible Resource}

In problems where a given resource is to be shared over time by consumers, the resource may be either fungible or non-fungible. Fungible resources are exemplified by the bandwidth allocation application: if the "bandwidth" in question is, say, the transfer rate provided for a connection in a computer network, the "identity" of the bandwidth allocated to a session is irrelevant (if it has any meaning at all). This type of bandwidth is therefore a fungible resource. Non-fungible resources are exemplified by real memory allocation in a multi-threaded programming environment, where chunks of memory are allocated and freed by the various threads. The total amount of memory allocated at any moment may not exceed the amount available, and in addition, the memory allocated to a thread must remain in the same position until freed. On top of the issue of fungibility, there is also the matter of contiguity. 
For example, a request to allocate memory can typically only be satisfied by a block of memory occupying contiguous addresses.

The general scheduling problem we have been dealing with so far was concerned with fungible resources. In this section we deal with contiguous allocation of a non-fungible resource. Specifically, we consider the problem of dynamic storage allocation (DSA). This problem models situations in which an allocation has to be contiguous (e.g., contiguous addresses in computer memory or a contiguous band of wavelengths in wireless communication) and cannot be changed until freed. The terminology we use is that of storage allocation, i.e., the resource is a contiguous storage area and activities are objects that require storage for different periods of time. We use the term width to refer to the size of an object, and denote by $W^{*}$ the maximum, taken over all times $t$, of the sum of the width demands at time $t$. The traditional goal in DSA has been to store all objects in minimum size storage. This problem is NP-Hard [Garey and Johnson 1979], but admits constant-factor polynomial-time approximation algorithms. In particular, Gergov [1999, 1996] and Kierstead [1991] describe "blow-up" algorithms that use a block of storage of size at most $\gamma \cdot W^{*}$, where $\gamma=3,5,6$, respectively. We use these algorithms to achieve approximation ratios of $\frac{1}{7}, \frac{1}{11}$, and $\frac{1}{18}$ (respectively) for the throughput maximization version of DSA (with profits associated with successful allocations of storage). In fact, we solve a more general problem in which a single request for storage may offer several alternative time intervals, only one of which is to be selected. The duration, storage demand, and profit may all vary among different intervals pertaining to the same request. This is just the bandwidth allocation problem with contiguity requirements thrown in. For this generalization, the performance guarantees we get are $\frac{1}{11}, \frac{1}{17}$, and $\frac{2}{55}$, respectively.

For simplicity, we normalize the amount of available storage to 1 . We partition the objects into wide objects, whose widths are more than $1 / 2$, and narrow objects, whose widths are at most $1 / 2$. We solve the bandwidth allocation problem separately for the wide objects and for the narrow objects: we obtain a $1 / 2$-approximation for the wide objects via interval scheduling, and a 1/3-approximation for the narrow objects as we have done for the bandwidth allocation problem. Let $S_{\mathrm{w}}$ be the solution for the wide objects and $S_{\mathrm{n}}$ the solution for the narrow objects. Observe that $S_{\mathrm{w}}$ is feasible for DSA since no two objects in it intersect. We proceed to extract from $S_{\mathrm{n}}$ a feasible solution for DSA, which we denote by $S_{\mathrm{n}}^{\prime}$, and return the solution with higher profit among $S_{\mathrm{w}}$ and $S_{\mathrm{n}}^{\prime}$.

We obtain $S_{\mathrm{n}}^{\prime}$ from $S_{\mathrm{n}}$ as follows. Given the objects in $S_{\mathrm{n}}$, we run any $\gamma$ "blow-up" algorithm and find a DSA solution that fits $S_{\mathrm{n}}$ in a storage block of size $\gamma \cdot W^{*}$, where $W^{*}$ is defined with respect to $S_{\mathrm{n}}$ (and therefore obeys $W^{*} \leq 1$ ). We partition this storage block into $\gamma$ non-overlapping strips, each of width $W^{*}$. Each such strip defines a feasible solution to our problem, consisting of the objects that are fully contained in the strip. Every object not fully contained in any of the strips crosses (exactly) one of the boundaries between two adjacent strips. Since no two objects crossing the same boundary intersect, and the objects are all narrow, each of the $\gamma-1$ boundaries defines a feasible solution that can be fit into a strip of width $1 / 2$. Thus any two of these solutions can be combined into a feasible solution to our problem. We choose $S_{\mathrm{n}}^{\prime}$ as the solution with maximum profit among the feasible solutions defined by the strips and the pairs of boundaries.

Denote by $p\left(S_{\mathrm{n}}\right)$ and $p\left(S_{\mathrm{n}}^{\prime}\right)$ the profits of $S_{\mathrm{n}}$ and $S_{\mathrm{n}}^{\prime}$, respectively. Then, either the total profit of the solutions defined by the strips is at least $2 \gamma p\left(S_{\mathrm{n}}\right) /(3 \gamma-1)$, 
and thus the best strip has profit at least $2 p\left(S_{\mathrm{n}}\right) /(3 \gamma-1)$, or else the total profit of the objects crossing boundaries is at least $p\left(S_{\mathrm{n}}\right)(\gamma-1) /(3 \gamma-1)$, and thus the best pair of boundaries have total profit at least $2 /(\gamma-1) \cdot p\left(S_{\mathrm{n}}\right)(\gamma-1) /(3 \gamma-$ $1)=2 p\left(S_{\mathrm{n}}\right) /(3 \gamma-1)$. Thus $p\left(S_{\mathrm{n}}^{\prime}\right) \geq 2 p\left(S_{\mathrm{n}}\right) /(3 \gamma-1)$. Since $p\left(S_{\mathrm{n}}\right)$ is a $1 / 3$ approximation for bandwidth allocation, $p\left(S_{\mathrm{n}}^{\prime}\right)$ is a $2 /(9 \gamma-3)$-approximation for bandwidth allocation. It is also a $2 /(9 \gamma-3)$-approximation for DSA since the optimum profit for DSA cannot be higher than the optimum for bandwidth allocation. Since either the optimum for the wide objects is at least $4 /(9 \gamma+1)$ of the optimum, or else the optimum for the narrow objects is at least $(9 \gamma-3) /(9 \gamma+1)$ of the optimum, the better solution among $S_{\mathrm{w}}$ and $S_{\mathrm{n}}^{\prime}$ is a $2 /(9 \gamma+1)$-approximation. Thus the "blow-up" algorithms of Gergov [1999, 1996] and Kierstead [1991] lead to approximation factors of $1 / 14,1 / 23$, and $2 / 55$, respectively.

It turns out, however, that the solutions returned by Gergov's algorithms [Gergov 1999; Gergov 1996] have the property that none of the objects cross strip boundaries ${ }^{1}$. Using similar arguments as above, the approximation factor can be tightened to $1 /(3 \gamma+2)$ for these algorithms. This yields approximation factors of $1 / 11$ and $1 / 17$, respectively.

In the special case where each request is limited to a single time interval (as is usually assumed in the DSA literature) we can do better still. Recall that in this special case, we were able to solve the bandwidth allocation problem optimally for wide jobs, and 1/2-approximately for narrow jobs. Therefore, the final ratio for DSA is $1 / 3 \gamma$ in this case, and for Gergov's algorithms it is $1 /(2 \gamma+1)$. Thus our best approximation ratio for this case is $1 / 7$, using the algorithm of Gergov [1999].

\section{Scheduling Sessions on Line and Ring Topologies}

As mentioned in the introduction, all of our results regarding the time axis interpretation hold for the processors axis interpretation as well. They hold for both throughput maximization and loss minimization. They also hold for the contiguous case. Moreover, since processors are discrete there are no issues of continuous "windows" to contend with (although it is hard to think of any "real" application involving activities with multiple instances in the context of connections between processors).

In this section we show how to adapt any approximation result for the throughput maximization problem on the line into one for the same problem on the ring. If the approximation factor for the line is $1 / \rho$, the resulting factor for the ring will be $1 /(\rho+1+\epsilon)$ where $\epsilon$ may be chosen arbitrarily small (the running time of the algorithm depends polynomially on $1 / \epsilon$ ).

The idea is to cut the ring at an arbitrary point and to partition the set of instances (of all the activities) into those that do not pass through the cut and those that do. We denote the former by $\mathcal{I}^{\prime}$ and the latter by $\mathcal{I}^{\prime \prime}$. The instances in $\mathcal{I}^{\prime}$ define an equivalent problem on the line, whereas the instances in $\mathcal{I}^{\prime \prime}$ amount to a knapsack problem. It is well known that the knapsack problem admits an FPTAS. We obtain a $1 / \rho$-approximation for $\mathcal{I}^{\prime}$ and a $1 /(1+\epsilon)$-approximation for $\mathcal{I}^{\prime \prime}$, and return the

\footnotetext{
${ }^{1}$ This is explicit in Gergov [1996] and is true for the algorithm in Gergov [1999] as well, even though it is not stated there explicitly [Gergov 2001].
} 
better solution of the two. Since either the optimum profit of the instances in $\mathcal{I}^{\prime}$ is at least $\frac{\rho}{\rho+1+\epsilon}$ of the optimum profit (for the original problem), or the optimum profit of the instances in $\mathcal{I}^{\prime \prime}$ is at least $\frac{1+\epsilon}{\rho+1+\epsilon}$ of the optimum, the the better solution is a $1 /(\rho+1+\epsilon)$ approximation.

\section{Appendix: A Primal-Dual Interpretation}

Local ratio algorithms can often be reformulated in primal-dual terms, and vice versa (see, e.g., Bar-Yehuda and Rawitz [2001]). We demonstrate this for our throughput maximization algorithm.

It turns out that the natural primal-dual formulation maps exactly onto our stackbased iterative algorithm of Section 3.3 only when every activity consists of a single instance. In all other cases it maps to a slightly modified version of our algorithm. (However, as pointed out in Bar-Yehuda and Rawitz [2001], our algorithm can be described in primal-dual terms as is, provided that we use a slightly different LP relaxation as the basis to the primal-dual description.) The modification required is to change the definition of $\mathcal{I}(\tilde{I})$ to the set of all instances intersecting $\tilde{I}$ (including $\tilde{I}$ itself and instances in $\mathcal{A}(\tilde{I})$ that intersect $\tilde{I})$. As a result of this change, the profit of each instance $I \in \mathcal{A}(\tilde{I}) \cap \mathcal{I}(\tilde{I})$ will now undergo two reductions. To prevent the profit of $\tilde{I}$ from becoming negative we must also scale the profit reductions by $1 /(1+\alpha w(\tilde{I}))$. Our performance guarantees hold for the modified algorithm as well, since the modification does not change the bound ratio $b_{\max } / b_{\mathrm{opt}}$.

Recall the integer programming formulation of the problem presented in Section 3. Let us construct the dual program of the linear relaxation of this program. We define $y_{i}$ as the dual variable corresponding to the constraint on activity $\mathcal{A}_{i}$ and $z_{t}$ as the dual variable corresponding to the constraint at time $t$. Let $T$ be the set of all start-times and end-times of all instances belonging to all activities and let $T_{I}=\{t \in T \mid s(I) \leq t<e(I)\}$ for all instances $I$.

$$
\text { Minimize } \sum_{i=1}^{m} y_{i}+\sum_{t \in T} z_{t}
$$

subject to:

For each activity instance $I \in \mathcal{A}_{i}: \quad y_{i}+w(I) \sum_{t \in T_{I}} z_{t} \geq p(I)$.

For all $i$ and $t$ :

$y_{i}, z_{t} \geq 0$.

In primal-dual terms, our algorithm proceeds as follows. We initially set all dual variables to zero. At each iteration the algorithm selects an instance $\widetilde{I} \in \mathcal{A}_{i}$ with minimum end-time among all instances whose corresponding dual constraints are violated. It pushes $\tilde{I}$ on the stack and increases $y_{i}$ by the amount $\delta$ required to make the constraint tight, and increases $z_{s}$ by $\alpha \delta$, where $s \in T$ is maximal such that $s<e(\tilde{I})$. When all dual constraints become satisfied, the algorithm constructs a schedule by popping each instance in turn off the stack and adding it to the schedule if doing so does not violate the schedule's feasibility.

Using the combinatorial properties of the solution, as we have done in our local ratio analysis of the various problems, we can charge at least $\delta$ units of profit to the 
profit $p(I)$ of some instance $I$ in the final solution for each increase of $(1+\alpha) \delta$ in the value of the dual objective function. Thus, the profit of the schedule is at least $1 /(1+\alpha)$ times the final value of the dual objective function and this is our performance guarantee.

ACKNOWLEDGMENT. We thank Sanjeev Khanna for many fruitful discussions and suggestions.

\section{REFERENCES}

AKCoglu, K., Aspnes, J., DAsGupta, B., AND KaO, M.-Y. 2002. Opportunity cost algorithms for combinatorial auctions. In Applied Optimization: Computational Methods in Decision-Making Economics and Finance, E. J. Kontoghiorghes, B. Rustem, and S. Siokos, Eds. Kluwer Academic Publishers. To appear.

AlbERS, S., ARORA, A., AND KhANNA, S. 1999. Page replacement for general caching problems. In 10th Annual ACM-SIAM Symposium on Discrete Algorithms (SODA). 31-40.

ARKIN, E. M., AND SILVERBERG, E. B. 1987. Scheduling jobs with fixed start and end times. Discrete Applied Mathematics 18, 1-8.

BAFNA, V., BERMAN, P., AND FuJITO, T. 1999. A 2-approximation algorithm for the undirected feedback vertex set problem. SIAM Journal on Discrete Mathematics 12, 289-297.

BAR-NoY, A., GuHA, S., NAOR, J., AND SCHIEBER, B. 2001. Approximating the throughput of multiple machines in real-time scheduling. SIAM Journal on Computing 31, 331-352.

BAR-YEHUDA, R. 2000. One for the price of two: a unified approach for approximating covering problems. Algorithmica 27, 131-144.

BAR-YeHUDA, R., AND EVEN, S. 1985. A local-ratio theorem for approximating the weighted vertex cover problem. Annals of Discrete Mathematics 25, 27-46.

BAR-YEHUDA, R., AND RAWITZ, D. 2001. On the equivalence between the primal-dual schema and the local-ratio technique. In 4th International Workshop on Approximation Algorithms for Combinatorial Optimization Problems (APPROX). Number 2129 in Lecture Notes in Computer Science. Springer, 24-35.

Berman, P., AND DasGupta, B. 2000. Multi-phase algorithms for throughput maximization for realtime scheduling. Journal of Combinatorial Optimization 4, 307-323.

ERLEBACH, T., AND JANSEN, K. 1998. Maximizing the number of connections in optical tree networks. In 9th Annual International Symposium on Algorithms and Computation (ISAAC). Number 1533 in Lecture Notes in Computer Science. Springer, 179-188.

ERLEBACH, T., AND JANSEN, K. 2000. Conversion of coloring algorithms into maximum weight independent set algorithms. In Satellite Workshops of the 27th International Colloquium on Automata, Languages, and Programming (ICALP), Workshop on Approximation and Randomization Algorithms in Communication Networks (ARACNE). 135-145.

GAREY, M., AND Johnson, D. 1979. Computers and Intractability: a Guide to the Theory of NP-Completeness. W.H. Freeman and Company.

GERGOV, J. 1996. Approximation algorithms for dynamic storage allocation. In 4th Annual European Symposium on Algorithms (ESA). Number 1136 in Lecture Notes in Computer Science. Springer, 52-61.

GERGOV, J. 1999. Algorithms for compile-time memory optimization. In 10th Annual ACM-SIAM Symposium on Discrete Algorithms (SODA). 907-908.

GERGOV, J. 2001. Personal communication.

Golumbic, M. 1980. Algorithmic graph theory and perfect graphs. Academic Press.

KhANNA, S. 1999. Personal communication.

KIERSTEAD, H. A. 1991. A polynomial time approximation algorithm for dynamic storage allocation. Discrete Mathematics 88, 231-237.

LEONARDI, S., MARChETti-SpaCCAMEla, A., AND VitaletTI, A. 2000. Approximation algorithms for bandwidth and storage allocation problems under real time constraints. In 20th Conference on Foundations of Software Technology and Theoretical Computer Science (FSTTCS). Number 1974 in Lecture Notes in Computer Science. Springer, 409-420.

PhILliPS, C. 2001. Personal communication.

PhILLIPS, C., UMA, R. N., AND WEIN, J. 2000. Off-line admission control for general scheduling problems. Journal of Scheduling 3, 365-381. 
SPIEKSMA, F. C. R. 1999. On the approximability of an interval scheduling problem. Journal of Scheduling 2, 215-227.

STEINBERG, A. 1997. A strip-packing algorithm with absolute performance bound 2. SIAM Journal on Computing 26, 401-409.

VAZIRANI, V. 1999. The primal-dual schema for approximation algorithms: where does it stand, and where can it go? Workshop on Approximation Algorithms for Hard Problems in Combinatorial Optimization, The Fields Institute for Research in Mathematical Sciences.

RECEIVED NOVEMBER 2000; REVISED JULY 2001; ACCEPTED JULY 2001 